\title{
Reflexiones sobre el patrimonio cultural de la salud en latinoamérica*
}

\author{
Reflections on cultural heritage of health \\ in latin america \\ Recibido: 06 de abril de 2010 - Revisado: 15 de abril de 2010 - Aceptado: 03 de mayo de 2010
}

Gabriela Campari** $^{* *}$

\section{Resumen}

El presente escrito analiza las experiencias e iniciativas dirigidas a promover el patrimonio cultural de la salud de Latinoamérica, con el objeto de facilitar medios adecuados que motiven el debate desde una lectura histórica y sociopolítica del espacio urbano, que amplíe la mirada para ver y entender la ciudad desde su paisaje.

Palabras clave

Patrimonio cultural, salud, paisaje, hospitales.

\begin{abstract}
This paper analyzes the experiences and initiatives to promote the cultural heritage of Latin America's health, in order to provide adequate means to motivate the debate from a historical and socio-political reading of urban space, to extend his eyes to see and understand the city from its landscape.
\end{abstract}

Keywords

Cultural heritage, health, landscape, hospital.

* Artículo de reflexión. El artículo

se encuentra inscrito en el marco del

proyecto de investigación de la Beca

Doctoral, financiado por la UBA

Secretaría de Ciencia y Técnica Re-

solución (CS) N. ${ }^{\circ}$ 5024/08 y en el

Programa de Doctorado Resolución

(CS) N. ${ }^{\circ} 469 / 08$, titulado: Paisajismo

y políticas públicas higienistas: el jar-

dín del hospital patrimonio cultural y

lugar de salud.

** Licenciada en Planificación y Di-

seño del Paisaje de la Universidad

de Buenos Aires (UBA), Argentina.

posgrado en Gestión Socio-Urbana y

Participación Ciudadana en Políticas

Públicas en la Facultad Latinoameri-

cana de Ciencias Sociales (FLACSO)

y en Gestión en Patrimonio Cultural

en la UBA. Docente-Investigadora

Universidad de Buenos Aires.

Correo electrónico:

gcampari@fadu.uba.ar //

gecampari@yahoo.com.ar 


\section{Introducción}

En la Conferencia Internacional de Promoción de la Salud, en la que se dio forma a la Carta de Bogotá (1992), se acordó asumir el compromiso de "estimular el diálogo de saberes diversos, de modo que el proceso de desarrollo de la salud se incorpore al conjunto del patrimonio cultural de la Región"1. A su vez, se hizo referencia al patrimonio cultural de América Latina dando cuenta de la responsabilidad que debían asumir los países de la región en las cuestiones de salud. En dicha dirección se impulsó la creación de la Biblioteca Virtual en Salud (BVS) Historia y Patrimonio Cultural de la Salud (HPCS) ${ }^{2}$, bajo la iniciativa de Brasil y Chile, con colaboración del Centro Latinoamericano y del Caribe de Información en Ciencias de la Salud,BIREME, la Organización Panamericana de la Salud, OPS y la Organización Mundial de la Salud, OMS, con el objeto de fortalecer y valorizar la historia y el patrimonio de la salud y promover la difusión pública y la conciencia social sobre su relevancia y desarrollo en los diversos ámbitos de gestión.

Según Susana Margulies "los procesos de la salud-enfermedad-atención constituyen una trama de saberes y de prácticas y abarcan el conjunto de nociones, categorizaciones y prescripciones que dan sentido y modelan las características de las prácticas sociales referidas a la enfermedad y a la muerte" $(2007$, p. 65$)$. La institucionalización de la medicina urbana, la noción de salubridad, las cuestiones referidas a la higiene y salud preventiva de la población, la problemática habitacional por el hacinamiento y las enfermedades contagiosas a nivel mundial promovieron transformaciones de lo urbano e impulsaron cambios de funcionalidades en diversos órdenes, siendo el campo arquitectónico de la salud uno de ellos, con edificios hospitalarios que sufrieron transformaciones en su concepción desde el tipo claustro, el pabellonal con jardines circundantes hasta las construcciones verticalizadas en bloque.
Desde esta perspectiva fue propuesta la BVS/ HPCS, haciendo hincapié en el reconocimiento y la importancia que revisten estos bienes para fortalecer la gestión del patrimonio cultural de la salud en sus dimensiones científicas, históricas y culturales.

Constituida la red se definieron seis campos de trabajo ${ }^{3}$, de los cuales tomamos el área de Patrimonio Arquitectónico con el objeto de presentar el marco contextual que dio origen a este modelo de gestión y a las iniciativas nacionales presentadas en la I Reunión de Coordinación Regional de la Red de la Biblioteca Virtual en Salud de Historia y Patrimonio Cultural de la Salud, a fin de reflexionar sobre las experiencias surgidas a partir del trabajo en red entre países latinoamericanos, abriendo el debate hacia otras miradas de construcción de identidades que integren la lectura histórica del patrimonio cultural de la salud en la construcción sociopolítica del espacio urbano y de los modos actuales de entender la ciudad.

\section{La Salud como patrimonio cultural}

La OMS definió la salud, en 1948, como el estado de completo bienestar físico, mental y social; aludiendo a la misma no sólo como ausencia de enfermedad sino como calidad de vida. La salud podía verse afectada -a favor o en detrimento- por diversos factores (políticos, económicos, sociales, culturales, de medio ambiente, de conducta y biológicos), resultando primordial promocionar los medios necesarios para mejorar y cuidar de su estado de bienestar ${ }^{4}$. A partir de la Conferencia Internacional de la UNESCO, en la cual se aprobó la Convención sobre la protección del patrimonio mundial, cultural y natural, el concepto de patrimonio cultural sentó precedente sobre la responsabilidad de los Estados en cuestiones patrimoniales $\mathrm{y}$, tal como se establece en el Art. $4^{\circ}$ de la Convención, la obligación de “(...) identificar, proteger, conservar, rehabilitar $\mathrm{y}$ transmitir a las generaciones futuras el patrimonio cultural y natural situado en su territorio (...)" (1972, p. 3). 
Dado este acuerdo, la promoción de la salud fue un tema recurrente en la agenda pública internacional, motivando la construcción de una serie de consideraciones que dieron origen a diversas Cartas y Declaraciones, ${ }^{5}$ las que pusieron en relieve la necesidad de instaurar un abordaje crítico en materia de salud pública. $\mathrm{Si}$ bien no forma parte del alcance de este trabajo analizar las aplicaciones de estas convenciones en América Latina, citamos ciertas características de la Carta de Ottawa (1986) y de Bogotá (1992) a fin de señalar antecedentes que dieron base a la integración de la salud en el patrimonio cultural. En la Carta de Ottawa se reconoció que "la salud se crea y se vive en el marco de la vida cotidiana; en los centros de enseñanza, de trabajo y de recreo. La salud es el resultado de los cuidados que uno se dispensa a sí mismo y a los demás, de la capacidad de tomar decisiones y controlar la vida propia y de asegurar que la sociedad en que uno vive ofrezca a todos sus miembros la posibilidad de gozar de un buen estado de salud" (Ottawa, 1986, p. 3). En este instrumento se identificaron cinco campos de acción:

a) Políticas públicas saludables;

b) Ambientes favorables a la salud;

c) Acción comunitaria;

d) Habilidades personales y

e) Reorientación del sistema de salud

que constituirían el andamiaje de trabajos llevados a cabo en conferencias internacionales posteriores, como la Carta de Bogotá sobre la promoción de la salud en América Latina, que definió como una de sus estrategias impulsar la cultura de la salud para promover los procesos sociales desde el compromiso social de los Estados, destacando su importancia como requisito prioritario para el desarrollo de América Latina. En este sentido, la cultura pasaba a ser un factor condicionante de salud, que requería estimular el diálogo entre los diversos saberes e integrarse en los procesos de desarrollo del patrimonio cultural. Si bien en algunos países de América se impulsaron diversas políticas para la salvaguarda del patrimonio cultural, las experiencias de Brasil y Chile sentaron antecedentes para el trabajo online entre los países de Latinoamérica.

La experiencia de Brasil en el campo patrimonial se potenció a partir del proceso de redemocratización, que posibilitó la restitución dederechos civilesy políticosy“(...) proporcionó condiciones favorables para numerosas acciones dirigidas a preservar parcelas significativas de la memoria y del patrimonio documental del país". (BVS/CRICS ${ }^{6}, 2005$, p. 5). Las primeras acciones de promoción de la salud se orientaron desde la historia, la memoria, la educación y la divulgación de las ciencias; rescatando antecedentes de la asistencia psiquiátrica, del movimiento sindical, de las entidades profesionales (sociedades médicas, academias) y de la producción científica de personajes célebres en la construcción social de la salud. En Chile, las cuestiones del patrimonio cultural de la salud comenzaron su desarrollo a través de las gestiones ministeriales, abordando el patrimonio desde la agenda pública, en acciones vinculadas con áreas "(...) como documentación, patrimonio arquitectónico, pesquisa histórica y difusión cultural, que afirman la historia y el patrimonio cultural como dimensiones relevantes de las políticas de salud en un contexto de reforma sectorial" (BVS/ CRICS, 2005, p. 7). En 2002 se creó la Unidad de Patrimonio Cultural de la Salud, actualmente dependiente de la División de Planificación Sanitaria de la Subsecretaría de Salud Pública del Ministerio de Salud, la que trabajó en una estrategia de acción que comprendía la creación de la Red Patrimonial, el fortalecimiento de las alianzas estratégicas intersectoriales y la definición y puesta en marcha de las áreas temáticas en relación al patrimonio cultural de la salud tangible/intangible. Este desarrollo se vio fortalecido con la constitución de la Red Latinoamericana de Historia y Patrimonio Cultural de la Salud (2005), conformada por el Ministerio de la Salud de Brasil/Casa de Oswaldo Cruz-Fiocruz y el Ministerio de la 
Salud de Chile/Unidad del Patrimonio Cultural de la Salud, con la colaboración de BIREME /OPS/OMS; dirigida a crear una biblioteca virtual que implementaría el comienzo de un trabajo mancomunado entre ambos países, a partir del compromiso de cooperación técnica asumido en estas temáticas.

Dos años más tarde, en 2007, tuvo lugar la I Reunión de Coordinación Regional de la Red de la Biblioteca Virtual en Salud de Historia y Patrimonio Cultural de la Salud (BVS/ HPCS), de la cual participaron representantes de la OPS/OMS y diez países de América Latina: Argentina, Brasil, Bolivia, Chile, Colombia, Costa Rica, Honduras, México, Paraguay y Uruguay. En dicho encuentro se definió al Patrimonio Cultural de la Salud como el "conjunto de bienes materiales y simbólicos socialmente construidos que expresan el proceso de la salud individual y colectiva en sus dimensiones científica, histórica y cultural"7. En la reunión, asimismo, se alcanzó consenso sobre algunas conclusiones y recomendaciones vinculadas a la difusión del patrimonio cultural de la salud, sustentadas en experiencias que estaban sucediendo en países de América Latina y el Caribe, y se acordó “(...) aumentar la visibilidad y los usos sociales del patrimonio cultural de la salud y contribuir así a su inserción en las políticas de protección social de la salud y en el desarrollo eficaz y equitativo de los sistemas nacionales de salud"8 $(2007$, p. 2).

La BVS/HPCS como espacio de gestión interactiva y de colaboración, fue concebida como un modelo en red de cooperación técnica entre las instituciones relacionadas con la historia y el patrimonio cultural de la salud de América Latina y el Caribe. Esta interacción permite compartir fuentes y flujos de información y conocimientos científicos, entendidos como bienes públicos nacionales, regionales y globales con acceso Web. El modelo aplica tres dimensiones: la primera es la referida a las redes sociales como construcciones colectivas en una trama de información científica y técnica en salud; catalogadas según ámbito geográfico, temáticas tratadas y procedencia institucional, desde una escala de circulación informativa de lo local a lo global. La segunda, hace referencia a los contenidos de la red como bienes públicos (colecciones, servicios y eventos, etc.) que generan fuentes y flujos de información, y la tercera dimensión, alude a los espacios participativos on-line en los que se generan redes de intercambio de información, experiencias y conocimientos, maximizando la inclusión de contenidos y espacios de colaboración para el patrimonio cultural de la salud. En este modelo de gestión se destaca la localización de sitios relevantes, seleccionados por su calidad y confiabilidad, en relación a la oferta en la red, que se incorporan como producciones "validadas" on-line. Si bien el modelo diversifica los canales informativos, la conformación de la red determina un recorte en las líneas direccionales de gestión y no sólo constituye "(...) un conjunto de herramientas, sino un entorno - un espacio, un ciberespacio- en el cual se producen las interrelaciones humanas" (Burbules y Callister, 2001, p. 19), que demandan una modalidad global en la comunicación y el tratamiento de la información. En este contexto, el patrimonio arquitectónico de la salud quedó definido por las edificaciones y espacios físicos que lo relacionan con la historia, la ciencia y la tecnología, que promocionan lagestión de acciones de identificación, registro y representación, con el objeto de ampliar y difundir el conocimiento de estos bienes patrimoniales.

\section{Visiones del patrimonio arquitectónico en salud}

La I Reunión de Coordinación Regional BVS/HPCS se caracterizó por presentar las visiones e iniciativas nacionales que se habían dado en lo patrimonial, haciendo referencia al estado de situación individual de los países intervinientes y las proyecciones esperadas del trabajo en red. El área de Patrimonio Arquitectónico estuvo relacionada íntimamente al campo de lo museológico y de lo hospitalario por las identificaciones y descripciones abordadas sobre el tema. La noción de patrimonio quedó ligada a 
un proceso social de nacionalismos, reconstruido por símbolos y personajes notables de la historia de la medicina, asociado a la herencia, al pasado y la tradición de la salud, que procuraba recuperar esencias y conformar identidades mediante el fortalecimiento de las redes sociales de la historia y del patrimonio cultural de la salud. La historia, por su profundidad temporal, y la estética, por lo bello o lujoso de lo construido, operaron como criterios de validación en la construcción social-política-económica de la salud. En este contexto patrimonial, en cuanto a lo arquitectónico, se mencionaron los casos del Instituto Médico Sucre y la Botica Hospital de Clínicas (Bolivia), Hospital San Juan de Dios (Costa Rica), Hospital General San Felipe (Honduras), Hospital de Clínicas (Paraguay) y el Lazareto de la Isla de Flores (Uruguay); bienes que, interpretados y promovidos, resultaron "seleccionados y activados" (Prats, 1996), despertando una suerte de susceptibilidad a ser considerados como patrimonio.

En las experiencias museológicas estuvo presente la idea de colección, de acumulación de objetos y bienes (obras de arte, muebles, estatuas, archivos, bibliografía, manuscritos, fotografías, mapas, etc.). Identificar, seleccionar y ordenar; donde el fichado pasaba a formar parte de la interpretación que se le había dado a lo arquitectónico para organizar las búsquedas (en red). El museo, desde su lugar de "templo de identidad" (Prats, 1996, p. 4), privilegió la representación patrimonial desde lo trascendental de la cultura y el lugar de resguardo más apropiado. En estos casos se mencionaron los museos in situ: Museo Virtual del Hospital Bernardino Rivadavia (Argentina), Museo de Historia de la Medicina-Hospital de Clínicas y Museo Anatomía-Instituto Médico Sucre (Bolivia), Museo de Historia de la Medicina-Facultad de Medicina Bogotá (Colombia), del nuevo Museo Nacional de Medicina Enrique Laval (Chile). A su vez, se citaron los museos que debieron readecuar la función a los edificios que los albergaban; a los que Juan Carlos Rico (1994) hace alusión como instituciones hospitalarias que resultan casos de difícil rehabilitación, donde el soporte de carácter histórico monumental condiciona la flexibilidad del uso expositivo ${ }^{9}$, mencionándose entre ellos: el Museo de Identidad Nacional -Antiguo Hospital General y Palacio de los Ministerios (Honduras)- y el Museo de la Medicina Mexicana -anteriormente Palacio de la Inquisición (México)-. Bajo esta primera mirada sobre las experiencias presentadas, pareciera que el estudio y la gestión se orientaron al mundo de los "expertos" y a ciertas comunidades interesadas en el tema. Retomando esta línea de análisis, María del Carmen Valdés Sagüés, haciendo referencia a lo museológico, que bien puede aplicarse al campo del patrimonio arquitectónico definido en la red, afirma que: "El museo tiene dos pilares esenciales, la colección y la sociedad. $\mathrm{Y}$ todas las tareas del museo tienen como objetivo relacionarlos entre sí con la finalidad de hacer comprensible la colección a la sociedad" (2008, p. 64). En este sentido, poner al alcance de la comunidad una propuesta de patrimonio cultural de la salud implica trabajar en la difusión de cuestiones que no resultan de simple entendimiento para un público ajeno o distante a estas temáticas. Por consiguiente, promocionar la educación desde el patrimonio arquitectónico implica fomentar gestiones que promuevan y amplíen la participación en la red de nuevas identidades.

\section{Conclusiones}

Si bien la tecnología informática presenta sus particularidades para ligar y acercar redes, multiplicando usuarios y miradas al tema, restaría pensar de qué modo podemos acortar esa brecha ante los tabúes y temores existentes en el imaginario social sobre las sensaciones discordantes entre la salud y el mal, el miedo al contagio o aquellas representaciones exógenas de la enfermedad analizadas por Francois Laplantine (1999), cuando son percibidas desde la acción de un elemento extraño que invade al cuerpo sano y lo agrede hasta, 
incluso, desencadenar la muerte. Ahora bien, ¿Cómo repensar la promoción del patrimonio cultural de la salud, sabiendo de los prejuicios y representaciones sociales que envuelve la enfermedad en los saberes populares? ¿Puede la sociedad encontrar deleite o disfrute en estos bienes patrimoniales y en su función cultural?

Resultará meritorio instalar nuevas discusiones sobre estas cuestiones; reflexionar sobre la custodia hospitalaria de los "objetos de colección", sin que dicha custodia represente el único medio de gestionar el patrimonio cultural de la salud; incluyendo el contexto histórico a partir de lecturas urbanas del paisaje que involucren el presente desde la mirada de las ciencias sociales, identificando la coexistencia de diferentes tiempos, yuxtapuestos e interactuantes, en un mismo espacio (Santos, 1988), con formas heterogéneas vinculadas a las diversas maneras de producir y construir lo urbano, en las cuales el tiempo y el espacio son vistos como categorías que funcionan unidas y forman parte de la dinámica ambiental, constribuyendo a promover la valoración de los lenguajes estéticos y sociales en la construcción del paisaje.

En esta línea de pensamiento, no resultaría ajeno abrir el debate sobre la idea del paisaje, con sus dinámicas urbanas y su organización espacial, en el cual se manifiesta el proceso de construcción ciudadana. En él se modeló la arquitectura a la que hoy reconocemos valor patrimonial, valor que, en cuanto a "lo hospitalario", no debiera acotarse a la idea de pieza de colección, sino incorporar las formas de ver y entender paisajísticamente la ciudad. En este sentido: ¿por qué no incorporar los aspectos que hicieron y hacen la construcción de las identidades y paisajes latinoamericanos? ¿Por qué no abordar el patrimonio cultural de la salud desde esta nueva mirada?

\section{Notas}

${ }^{1}$ Declaración de la Conferencia Internacional de Promoción de la Salud-Promoción de la Salud en América Latina-Santa Fe de Bogotá, noviembre de 1992.

${ }^{2}$ www.bvsalud.org

${ }^{3}$ Referidos a: Literatura científica y técnica; Colección de textos completos; Directorios; Personajes; Patrimonio arquitectónico, archivístico y museológico e Investigaciones históricas en salud.

${ }^{4} 1^{\circ}$ Conferencia Internacional sobre la Promoción de la Salud reunida en Ottawa el 21 de noviembre de 1986.

${ }^{5}$ En torno a la aplicación estratégica de la Carta de Ottawa tuvieron lugar: Adelaide (1988), políticas públicas saludables; Sundsvall (1991); Carta de Bogotá (1992), que se desarrolla en los párrafos posteriores; Carta del Caribe (1993), las habilidades personales fortaleciendo la comunicación social y la educación en salud; Declaración de Yakarta (1997) sobre la acción comunitaria; Declaración de México (2000), guía de propuesta de plan de acción; y la Carta de Bangkok (2005), en la cual se plantearon cuatro compromisos claves para la promoción de la salud: 1. integre la agenda de desarrollo mundial, 2. una responsabilidad esencial de todos los niveles de gobierno, 3. un objetivo fundamental de las comunidades y la sociedad civil y 4. un requisito de las buenas prácticas empresariales.

${ }^{6} 7^{\circ}$ Congreso Regional de Información en Ciencias de la Salud.

${ }^{7}$ Grupo de Trabajo Historia y Salud de la $4^{\circ}$ Reunión de Coordinación Regional de Bibliotecas Virtuales en Salud (BVS) y el $7^{\circ}$ Congreso Regional de Información en Ciencias de la Salud (CRICS).Términos de Constitución de la Red de Historia y Patrimonio Cultural de la Salud. Salvador Bahía, Brasil 2005.

${ }^{8}$ Presentación y programa en: http://www. eventos.bvsalud.org/BVSHPCS/presentation php?lang=es 
9 Observación que no escapa de ser considerada para los museos in situ.

\section{Referencias}

Burbules, N. y Callister, T. (2001). Educación: riesgos y promesas de las nuevas tecnologías. Editorial Granica: Barcelona.

BVS y CRICS (2005). Términos de Constitución de la Red de Historia y Patrimonio Cultural de la Salud. $7^{\circ}$ Congreso Regional de Información en Ciencias de la Salud. Salvador Bahía, Brasil. Recuperado el 20 de enero de 2010, de: http://www. bvshistoria.coc.fiocruz.br/hpcs/termconsti-tuicaorhpcses.pdf.

BVS/HPCS (2007). I Reunión de Coordinación Regional. Conclusiones y Recomendaciones. Del 1 al 3 de agosto de 2007. Santiago, Chile. Recuperado el 15 de diciembre de 2009, de http://www.eventos.bvsalud.org/BVSHPCS/presentation php?lang=es.

Laplantine, F. (1999). La antropología de la enfermedad. Serie Antropológica, Buenos Aires: Ediciones del Sol.

Margulies, S. (2008). Cultura y salud. Una perspectiva antropológica. En: Sipes, Celia (comp.). Patrimonio Cultural Hospitalario. Temas de Patrimonio Cultural N. ${ }^{\circ}$ 21 (pp: 63-69). Comisión para la preservación del Patrimonio Histórico Cultural Gobierno de la Ciudad de Buenos Aires. Buenos Aires.

OMS (1998). Promoción de la salud. Glosario. Recuperado el 18 de diciembre de 2009, de http://www.msc.es/profesionales/salud Publica/prevPromocion/docs/glosario. pdf.
OMS/OPS (1986). Carta de Ottawa para la Promoción de la Salud. Recuperado el 18 de diciembre de 2009, de http://www.paho. org/Spanish/AD/SDE/HS/OttawaCharter Sp.pdf.

OMS/OPS (2006). Promoción de la salud: logros y enseñanzas extraídas entre La Carta de Ottawa y la Carta de Bangkok $y$ perspectivas para el futuro. En: $138^{\mathrm{a}}$ Sesión del Comité Ejecutivo. Del 19 al 23 de junio de 2006. Punto 4.8 del orden del día provisional CE138/16 (Esp.) 2 de junio de 2006. Washington, D.C., Estados Unidos.

OPS (1992). Declaración de la Conferencia Internacional de Promoción de la Salud -Promoción de la Salud en América Latina- Santa Fe de Bogotá. Recuperado el 12 de diciembre de 2009, de http://www. bvsde.paho.org/bvsacd/cd51/promocionbogota.pdf.

Prats, L. (1996). El quehacer de los antropólogos. Barcelona: Editorial Ariel

Rico, J. C. (1994). Museos, arquitectura y arte. Madrid: Sílex Ediciones,

Santos, M. (1996). Metamorfosis del espacio habitado. Barcelona: Oikos-Tau.

Unesco (1972). Convención sobre la protección del patrimonio mundial, cultural y natural. París, 16 de noviembre de 1972. Recuperado el 03 de enero de 2010, de http://whc.unesco.org/archive/convention-es.pdf.

Valdés Sagüés, M. C. (2008). La difusión, una función del museo. En: museos.es, revista N. ${ }^{\circ}$ 4. (pp: 64-75). Ministerio de Cultura, Museos Estatales, Madrid. 
